\title{
Phase Transition in Continuum Potts Models
}

\author{
H.-O. Georgii ${ }^{1, \star}$, O. Häggström ${ }^{2, \star \star}$ \\ ${ }^{1}$ Mathematisches Institut der Universität München, Theresienstr 39, D-80333 München, Germany. \\ E-mail: georgii@rz.mathematik.uni-muenchen.de \\ ${ }^{2}$ Dept of Mathematics, Chalmers University of Technology, 41296 Göteborg, Sweden \\ E-mail: olleh@math chalmers.se
}

Received: 25 February 1996 / Accepted: 21 April 1996

To the memory of Roland Dobrushin

\begin{abstract}
We establish phase transitions for a class of continuum multi-type particle systems with finite range repulsive pair interaction between particles of different type. This proves an old conjecture of Lebowitz and Lieb. A phase transition still occurs when we allow a background pair interaction (between all particles) which is superstable and has sufficiently short range of repulsion. Our approach involves a random-cluster representation analogous to the Fortuin-Kasteleyn representation of the Potts model. In the course of our argument, we establish the existence of a percolation transition for Gibbsian particle systems with random edges between the particles, and also give an alternative proof for the existence of Gibbs measures with superstable interaction.
\end{abstract}

\section{Introduction}

Although the study of phase transitions for Gibbsian systems is one of the main subjects of statistical mechanics, examples of models exhibiting phase transition are mainly restricted to lattice systems. For systems of particles in the continuum the situation is still quite unsatisfactory. Besides the mere existence of phase transitions for some unknown interactions (as shown in Appendix B of Israel [17]) and the canonical-ensemble approach of Johansson $[18,19]$ in one dimension, there exists essentially only one specific model for which a phase transition is known to occur: the model of Widom and Rowlinson [32]. This is a multi-type particle system in $\mathbb{R}^{d}, d \geq 2$, with hard-core exclusion between particles of different type, and no interaction between particles of the same type. The phase transition in this model for large activities $z$ was established by Ruelle [31] using a version of the Peierls argument. Lebowitz and Lieb [23] extended his result to multi-type particle systems in which the hard-core exclusion is replaced by a soft-core repulsion between unlike particles. Such models can also be viewed as continuum versions of the Ising or Potts model. Lebowitz and Lieb needed the condition that the soft-core repulsion

\footnotetext{
* Research partially supported by the Isaac Newton Institute Cambridge.

$\star \star$ Research supported by the Swedish Natural Science Research Council and the Deutsche Forschungsgemeinschaft
} 
is sufficiently strong (or, equivalently, that the temperature is sufficiently low), and conjectured that a phase transition for large $z$ should still occur without this proviso

In this paper we establish the existence of phase transitions for a large class of continuum Potts models including the Widom-Rowlinson model and its soft-core variant. In particular, we shall verify the preceding conjecture. We also include a background interaction between all particles (independently of their types) which is superstable but not too repulsive in relation to the intertype repulsion. Our approach is based on a random-cluster representation analogous to the Fortuin-Kasteleyn representation of the lattice Potts model, see $[8,7,16]$. This gives a certain continuum percolation process, which has previously been studied e.g. by Given and Stell [15] from a different point of view. A phase transition for the continuum Potts model follows if we can show that the corresponding percolation process contains an infinite cluster A similar program was recently carried out by Chayes et al. [3] for the hardcore Widom-Rowlinson model (related ideas appear also in [14]). In that case, the existence of infinite clusters follows from a stochastic comparison with the Poisson Boolean model of continuum percolation (see [25]), while our more general framework requires somewhat more involved arguments. We note that the random-cluster representation requires the symmetry of interaction between particles of different types In non-symmetric Widom-Rowlinson models, the existence of a phase transition has been established by Bricmont et al. [2] using a version of the Pirogov-Sinai theory.

We now describe the setup. We consider the Euclidean space $\mathbb{R}^{d}, d \geq 2$, and fix an integer $q \geq 2$. Let . $x$; denote the class of all locally finite subsets of $\mathbb{K}^{d} \mathrm{~A}$ configuration of particles in $\mathbb{R}^{d}$ with $q$ distinct types (without multiple occupancies) is described by a vector $\mathbf{X}=\left(X_{1}, \quad, X_{q}\right)$ of pairwise disjoint sets $X_{1}, \quad, X_{q} \in X^{\prime}$ : We write $\mathscr{X}^{\cdot(q)}$ for the class of all such multitype configurations Any $\mathbf{X} \in \cdot \mathscr{X}^{\cdot(q)}$ is uniquely determined by the pair $(X, \sigma)$, where $X=\operatorname{sp}(\mathbf{X}):=X_{1} \cup \ldots \cup X_{q}$ is the set of all occupied positions, and the type function $\sigma=\sigma(\mathbf{X}): X \rightarrow\{1, \quad, q\}$ is defined by $\sigma(x)=s$ if $x \in X_{s}, 1 \leq s \leq q$. $x^{*}$ is equipped with the $\sigma$-algebra which is generated by the counting variables $x^{\circ} \ni X \rightarrow \#(X \cap \Delta)$ for bounded measurables $\Delta \subset \mathbb{R}^{d}$, and $\cdot \mathscr{X}^{\cdot(q)}$ with the restriction of the product $\sigma$-algebra on $\mathscr{X}^{*}$. . The set of all configurations in a measurable set $\Lambda \subset \mathbb{R}^{d}$ will be denoted by $\mathscr{X}_{\Lambda}^{(q)}$, and the corresponding $\sigma$-algebra is similarly defined

The interaction between the particles is assumed to be given by a formal Hamiltonian of the form

$$
\begin{aligned}
H(\mathbf{X}) & =H^{\varphi}(\mathbf{X})+H^{\psi}(X) \\
& =\sum_{\substack{\{x, y\} \subseteq X \\
\sigma(x) \neq \sigma(y)}} \varphi(x-y)+\sum_{\{x, y\} \subseteq X} \psi(x-y)
\end{aligned}
$$

Here $\mathbf{X} \in \mathscr{X}^{*}(q), X=\operatorname{sp}(\mathbf{X}), \sigma=\sigma(\mathbf{X})$, and the pair potentials $\left.\left.\varphi, \psi \cdot \mathbb{R}^{d} \rightarrow\right]-\infty, \infty\right]$ are even measurable functions. The first term $H^{\varphi}$ is the most important contribution to the Hamiltonian. It describes a repulsion between particles of different types. The second term $H^{\psi}$ corresponds to a type-independent background pair interaction Specifically, we assume the following There exist constants $u>0$ and $0 \leq r_{1} \leq r_{2}<r_{3} \leq r_{4}$ such that

(A1) (strict repulsion of $\varphi$ ) $\quad \varphi \geq 0$, and $\varphi(x) \geq u$ when $|x| \leq r_{3}$.

(A2) (finite range of $\varphi$ ) $\quad \varphi(x)=0$ when $|x| \geq r_{4}$. 
(A3) (strong stability and regularity of $\psi$ ) either $\psi \geq 0$, or $\psi$ is superstable and lower regular in the sense of Ruelle [30], see (13) and (14) below.

(A4) (short range of repulsion for $\psi$ ) $\quad \psi(x) \leq 0$ when $|x| \geq r_{2}$, and the positive part $\psi_{+}$of $\psi$ satisfies

$$
\int_{\left\{|x| \geq r_{1}\right\}} \psi_{+}(x) d x<\infty .
$$

(A5) (scale relations) $r_{2}<r_{3} / 2 \sqrt{d+3}$, and $r_{1}$ is sufficiently small (depending on $q, u, r_{2}, r_{3}$; cf. (11) below).

We believe that already the case $\psi \equiv 0$ is of some interest. The reader has the option of concentrating on this case and forgetting about conditions (A3)-(A5); he then can skip quite a number of technicalities in Sects. 3 and 4.

Given a finite box $\Lambda \subset \mathbb{R}^{d}$, a boundary condition $\mathbf{Y} \in \mathscr{C}_{\Lambda^{c}}^{(q)}$, and an activity parameter $z>0$, the associated Gibbs distribution $Q_{\Lambda \mid \mathbf{Y}}$ on $\mathscr{C}_{\Lambda}^{(q)}$ is defined by

$$
Q_{\Lambda \mid \mathbf{Y}}(d \mathbf{X})=Z_{\Lambda \mid \mathbf{Y}}^{-1} \exp \left[-H_{\Lambda \mid \mathbf{Y}}(\mathbf{X})\right] \pi_{\Lambda}\left(d X_{1}\right) \ldots \pi_{\Lambda}\left(d X_{q}\right) .
$$

In the above,

$$
\begin{aligned}
H_{\Lambda \mid \mathbf{Y}}(\mathbf{X}) & =H_{\Lambda \mid \mathbf{Y}}^{\varphi}(\mathbf{X})+H_{\Lambda \mid Y}^{\psi}(X) \\
& =\sum_{\substack{\{x, y\} \subseteq X \cup Y Y \\
\{x, y\} \cap \Lambda \rightarrow \emptyset, \sigma(x) \neq \sigma(y)}} \varphi(x-y)+\sum_{\substack{\{x, y\} \subseteq X \cup Y \\
\{x, y\} \cap \Lambda \neq \emptyset}} \psi(x-y)
\end{aligned}
$$

[with $\mathbf{X} \in \mathscr{C}_{\Lambda}^{(q)}, X=\operatorname{sp}(\mathbf{X}), Y=\operatorname{sp}(\mathbf{Y})$, and $\sigma$ the common extension of $\sigma(\mathbf{X})$ and $\sigma(\mathbf{Y})]$ is the Hamiltonian in $\Lambda$ with boundary condition $\mathbf{Y} ; \pi_{\Lambda}=\pi_{\Lambda}^{z}$ is the Poisson point process on $\mathscr{C}_{\Lambda}$ with constant intensity $z$, i.e.

$$
\int f d \pi_{\Lambda}=e^{-z|\Lambda|} \sum_{n=0}^{\infty} \frac{z^{n}}{n !} \int_{\Lambda^{n}} f\left(\left\{x_{1}, \ldots, x_{n}\right\}\right) d x_{1} \ldots d x_{n}
$$

for any bounded measurable function $f$ on $\mathscr{C}_{\Lambda}$; and $Z_{\Lambda \mid \mathbf{Y}}$ is a normalizing constant usually called the partition function. (We will always use symbols like $Z_{\Lambda \mid \mathbf{Y}}$ to denote such normalizing constants, but their precise meaning will vary with the context. Also, throughout the paper we will often suppress the dependence of some quantities on the parameters $z, q, \varphi, \psi$ which will be considered as fixed.) Later on, we will prefer to consider $Q_{\Lambda \mid \mathbf{Y}}$ as a measure on $\mathscr{C}^{(q)}$ which is supported on the set $\mathscr{C}_{\Lambda \mid \mathbf{Y}}^{(q)}=\{\mathbf{X} \in$ $\mathscr{C}^{(q)}: X_{i} \backslash \Lambda=Y_{i}$ for $\left.1 \leq i \leq q\right\}$.

Since $\psi$ is not necessarily of finite range, $Q_{\Lambda \mid \mathbf{Y}}$ is only well-defined if we require that $\mathbf{Y}$ (resp. its support $Y$ ) is tempered in the sense that

$$
\sup _{k \geq 1} k^{-d} \sum_{i \in \mathbf{Z}^{d}:|i| \leq k} N_{i}(\mathbf{Y})^{2}<\infty
$$

where $N_{i}(\mathbf{Y})=\#(\mathbf{Y} \cap \Delta(i))$ is the number of particles in the cubic cell

$$
\Delta(i)=\left[-\delta / 2, \delta / 2\left[^{d}+\delta i, \quad i \in \mathbb{Z}^{d},\right.\right.
$$

of an arbitrarily fixed side length $\delta>0$. It is then easy to see from (13) and (14) that $H_{\Lambda \mid \mathbf{Y}}$ is well-defined and $\exp \left[-H_{\Lambda \mid \mathbf{Y}}\right]$ is integrable with respect to $\left(\pi_{\Lambda}\right)^{q}$. 
It is evident from (1) that, for fixed $X \in \cdot X_{\Lambda}$, the conditional distribution of $\sigma(\mathbf{X})$ under the condition $\mathrm{spX}=X$ relative to $Q_{\Lambda \mid \mathbf{Y}}$ is that of a discrete Potts model on $X$ with a position-dependent interaction between the type-variables. This justifies calling our model a continuum Potts model

Definition. A probability measure $Q$ on $\mathscr{X}^{\cdot(q)}$ is called a tempered Gibbs measure for the continuum Potts model or, for short, a continuum Potts measure with activity $z>0$ and interaction potentials $\varphi, \psi$ if $Q$ is supported on the set of tempered configurations and, for any box $\Lambda \subset \mathbb{R}^{d}$, the probability kernel $\mathbf{Y} \rightarrow Q_{\Lambda \mid \mathbf{Y}}$ from. $\mathscr{X}_{\Lambda^{c}}^{(q)}$ to $\mathscr{X}_{\Lambda}^{\cdot(q)}$ is a version of the conditional distribution under $Q$ of the configuration in $\Lambda$ given the configuration outside of $\Lambda$.

Continuum Potts measures always exist, as will be shown in Sect. 4. A phase transition is said to occur if there exists more than one continuum Potts measure. The following theorem, which is our main result, shows that this happens when $z$ is sufficiently large.

Theorem 1.1. Suppose $\varphi$ and $\psi$ satisfy assumptions (Al) to (A5), and let $q \geq 2$. If the activity $z$ is sufficiently large (depending on the model parameters $d, u$, and $r_{1}$ to $\left.r_{4}\right)$, there exist at least $q$ distinct continuum Potts measures for $z, q, \varphi, \psi$ which are invariant and ergodic under translations and can be distinguished by the densities of particles with a fixed type.

In Remark 4.3 at the end of the paper we will show that the phase transition manifests itself also thermodynamically by a non-differentiablity ("discontinuity") of the pressure. Here is a number of further remarks.

Remark 1.2. The scale assumptions (A5), in their present form, are certainly an artefact of our method. We note, however, that the condition $r_{1}<r_{3}$ is essential. For if $\psi$ has a hard core of radius $\geq r_{3}$ then the particles do not feel the additional intertype repulsion of range $r_{3}$.

Remark 1.3. As a kind of converse of Theorem 1.1, we have that the continuum Potts measure is unique for sufficiently small $z$ (depending on $\varphi, \psi$ and $q$ ). This can be deduced from (A3) either by the method of correlation equations [27, 30] or, under a slight sharpening of (A3), by Dobrushin's uniqueness criterion $[4,5]$. This leads naturally to the question of whether the occurrence of phase transition is monotone in $z$, i.e., whether there exists a critical $z_{c}=z_{c}(d, q, \varphi, \psi)$ such that uniqueness holds for $z<z_{c}$ and non-uniqueness for $z>z_{c}$. We are unable to settle this issue

Remark 1.4. In the above, the inverse temperature $\beta$ was set equal to 1 This is no loss of generality because $\beta$ can be absorbed into $\varphi$ and $\psi$. However, one may ask for the phase transition region in the $(z, \beta)$-plane when $q, \varphi, \psi$ are fixed. As the proof of Theorem 11 will show, there exists a decreasing function $\left.z_{0}:\right] 0, \infty[\rightarrow] 0, \infty[$ (depending on all model parameters) such that the continuum Potts measure is nonunique whenever $\beta>0$ and $z>z_{0}(\beta)$. In particular, for given $z>z_{0}(\infty)$ a phase transition occurs for sufficiently large $\beta$, but as in the previous remark we are unable to answer the question of monotonicity in $\beta$

Remark 1.5 Suppose we take $q=2$ and $\psi \equiv 0$ and consider, for each $\mathbf{X} \in \mathscr{X}^{\cdot(2)}$, the set $X_{1} \in \mathscr{X}^{\circ}$ of particles of type 1 . Let $\Lambda \subset \mathbb{R}^{d}$ be a fixed box and $\Delta \supset \Lambda$ so large that the distance between $\Lambda$ and $\mathbb{R}^{d} \backslash \Delta$ exceeds the range $r_{4}$ of $\varphi$. It is easy to see 
that the conditional distribution of $X_{1} \cap \Lambda$ given $X_{1} \backslash \Lambda$ under $Q_{\Delta \mid \mathbf{Y}}$ does not depend on $\mathbf{Y}$ and has a $\pi_{\Lambda}$-density proportional to

$$
\begin{aligned}
& \int \pi_{\Delta}\left(d X_{2}\right) \exp \left[-\sum_{y \in X_{2}} \sum_{x \in X_{1}} \varphi(x-y)\right] \\
& =\exp \left[-z \int\left(1-\exp \left[-\sum_{x \in X_{1}} \varphi(x-y)\right]\right) d y\right] .
\end{aligned}
$$

The image of a continuum Potts measure under the projection $\mathbf{X} \rightarrow X_{1}$ is therefore a Gibbs measure on $\mathscr{C}$ for the formal Hamiltonian

$$
H_{z}^{(1)}(X)=z \int\left(1-\exp \left[-\sum_{x \in X} \varphi(x-y)\right]\right) d y
$$

and the activity $z$. In the special case of the hard-core potential

$$
\varphi(x)= \begin{cases}\infty & \text { for }|x| \leq r \\ 0 & \text { otherwise }\end{cases}
$$

this relation between the two-type model and its one-type marginal was discovered by Widom and Rowlinson [32]. We conclude from Theorem 1.1 that, for large $z$, the one-type particle system with Hamiltonian $H_{z}^{(1)}$ and activity $z$ exhibits a phase transition, in that there exist two translation-invariant Gibbs measures with different particle densities.

The rest of this paper is organized as follows. In Sect. 2, we obtain the randomcluster representation for the continuum Potts model on a compact set $\Lambda \subset \mathbb{R}^{d}$. Section 3 is devoted to the existence of percolation in the random cluster model and, in particular, for Gibbsian systems of particles of a single type. By "percolation" we here mean the occurence of infinite clusters. In Sect. 4 we conclude the proof of Theorem 1.1 by taking the infinite-volume limit $\Lambda \uparrow \mathbb{R}^{d}$. In particular, we obtain the existence of continuum Potts measures for arbitrary $z>0$ and thus, in the case $q=1$, the existence of tempered Gibbs measures for pair interactions $\psi$ satisfying (A3) and a condition of upper regularity that weakens (A4).

\section{The Random-Cluster Representation}

The purpose of this section is to obtain a joint construction of the continuum Potts model on a fixed finite box $\Lambda \subset \mathbb{R}^{d}$ with parameters $z, \varphi$ and $q$, and a related edge process which we call the continuum random-cluster model. This is analogous to the joint construction of the discrete Potts model and its Fortuin-Kasteleyn representation, which was discovered by Edwards and Sokal [7]. The continuum random-cluster model was introduced first by Klein [22]; see [15] and the references there for some subsequent work. In the particularly nice case of the Widom-Rowlinson model when $\psi \equiv 0$ and $\varphi$ is given by (4), the random-cluster representation was rediscovered independently by Chayes et al. [3] and by the present authors.

In the course of the construction, we will pay particular attention to measurability questions. One reason for doing so is that some of the earlier work on continuum percolation involves constructions of probability spaces in which most of the interesting events turn out not to be measurable, as pointed out by Meester and Steif $[26,25]$. 
Let $Y \in \mathscr{X}_{\Lambda^{c}}$ be a fixed tempered boundary configuration. We identify $Y$ with the boundary condition $\mathbf{Y}=(Y, \emptyset, \quad, \emptyset) \in \mathscr{X}_{\Lambda^{c}}^{(q)}$ of type-1 particles. (The assumption of constant type might seem unnaturally restrictive, but it keeps things simpler and is sufficient for our purposes.)

The basic idea of the random-cluster representation is to introduce random edges between the particle positions We thus introduce the set

$$
E_{\mathbb{R} \mathbb{R}^{d}}=\left\{e=\{x, y\} \subset \mathbb{R}^{d}: x \neq y\right\}
$$

of all possible edges between pairs of points in $\mathbb{R}^{d}$. The natural $\sigma$-algebra on $E_{\mathbb{R}}{ }^{d}$ consists of all sets of the form $\left\{\{x, y\} \in E_{\mathbb{R}^{d}}:(x, y) \in A\right\}$ with measurable $A \subseteq$ $\mathbb{R}^{d} \times \mathbb{R}^{d}$. In particular, for any measurable $\Delta \subseteq \mathbb{R}^{d}$ the set

$$
E_{\Delta}=\left\{e \in E_{\mathbb{R}^{d}} \cdot e \subseteq \Delta\right\}
$$

of all edges within $\Delta$ is measurable. We write

$$
\mathscr{\zeta}=\left\{E \subset E_{\mathbb{R} d}: E \text { locally finite }\right\}
$$

for the set of all edge configurations. \& comes equipped with the $\sigma$-algebra which is generated by the counting variables $E \rightarrow \#(E \cap F)$ with bounded measurable $F \subset E_{\mathbb{R}}$.

We will construct a probability measure $\mathbb{P}=\mathbb{P}_{\Lambda \mid Y}^{z q}$ on $\mathscr{X}^{(q)} \times \mathscr{S}$ which models the following random mechanism. Indistinguishable particles appear in $\Lambda$ according to a Gibbs distribution for the background interaction $\psi$ with activity $z q$ and boundary condition $Y$. Each particle picks a type at random from $\{1, \quad, q\}$. Finally, each pair $e=\{x, y\}$ of particles (one of which may belong to $Y$ ) is connected by an edge with probability $p(e)=1-\exp [-\varphi(x-y)]$, independently of all other pairs; the particles in $Y$ are all connected to each other deterministically. Note that $\varphi$ enters the construction only via the edge probabilities $p(e)$.

Specifically, the measure $\mathbb{P}$ will be built from the following three constituents.

- The distribution of particle positions. This is given by the (single-type particle) Gibbs distribution $P_{\Lambda \mid Y}^{z q}$ in $\Lambda$ with boundary condition $Y$, interaction $\psi$, and activity $z q$, i.e.,

$$
P_{\Lambda \mid Y}^{z q}(d X)=Z_{\Lambda \mid Y}^{-1} \exp \left[-\sum_{\{x, y\} \in E_{X \cup Y} \backslash E_{Y}} \psi(x-y)\right] \pi_{\Lambda}^{z q}(d X),
$$

$X \in \mathscr{X}_{\dot{\Lambda}}$ (Note that this measure coincides with $Q_{\Lambda \mid \mathbf{Y}}$ when $q=1$.) Henceforth we shall view $P_{\Lambda \mid Y}^{z q}$ as a probability measure on $\mathscr{X}^{\circ}$ which is supported on the set $\cdot X_{\Lambda \mid Y}=\{X \in \mathscr{X}: X \backslash \Lambda=Y\}$

- The type picking mechanism. For a fixed set of positions $X \in \mathscr{X}_{\Lambda \mid Y}$, we let $\lambda_{X, \Lambda}$ denote the distribution of the random vector

$$
\left(\left\{x \in X: \tau_{x}=s\right\}\right)_{1 \leq s \leq q} \in \cdot \mathscr{X} \cdot(q),
$$

where $\left(\tau_{x}\right)_{x \in X \cap \Lambda}$ are independent and uniformly distributed on $\{1, \quad, q\}$, whereas $\tau_{x}=1$ for $x \in X \backslash \Lambda=Y$. 
- The edge drawing mechanism. For given $X \in \mathscr{K}_{\Lambda \mid Y}$, let $\mu_{X, \Lambda}$ denote the distribution of the random edge configuration $\left\{e \in E_{X}: \eta_{e}=1\right\} \in \mathscr{E}$, where $\left(\eta_{e}\right)_{e \in E_{X}}$ are independent $\{0,1\}$-valued random variables with

$$
\operatorname{Prob}\left(\eta_{e}=1\right)=p_{\Lambda}(e):=\left\{\begin{array}{cl}
1-e^{-\varphi(x-y)} & \text { when } e=\{x, y\} \in E_{\mathbb{R}^{d}} \backslash E_{\Lambda^{c}} \\
1 & \text { when } e \in E_{\Lambda^{c}}
\end{array}\right.
$$

The measure $\mu_{X, \Lambda}$ is a point process on $E_{\mathbb{R}^{d}}$ (which may be viewed as a $p_{\Lambda^{-}}$ thinning of the complete edge set $E_{X}$ ).

The desired probability measure $\mathbb{P}$ on $\mathscr{K}^{(q)} \times \mathscr{E}$ is now defined by

$$
\mathbb{P}=\mathbb{P}_{\Lambda \mid Y}^{z q}:=\int P_{\Lambda \mid Y}^{z q}(d X) \lambda_{X, \Lambda} \otimes \mu_{X, \Lambda}
$$

This definition makes sense because the mappings $X \rightarrow \lambda_{X, \Lambda}$ and $X \rightarrow \mu_{X, \Lambda}$ are probability kernels, as we will show in Lemma 2.4 below. IP is supported on the set of all $(\mathbf{X}, E) \in \mathscr{C}^{(q)} \times \mathscr{E}$ which are such that $\mathbf{X} \in \mathscr{C}_{\Lambda \mid \mathbf{Y}}^{(q)}$ and $E \subseteq E_{X}$ with $X=\operatorname{spX}$.

We now consider the event $\Omega \subset \mathscr{B}^{(q)} \times \mathscr{E}$ that the particle types are constant on each connected component of the graph $\left(\mathrm{sp} \mathbf{X}, E \cap E_{\mathrm{sp} \mathbf{X}}\right)$. Equivalently, $\Omega$ can be described as the event that no two vertices with different types have an edge in common. Formally, we can write

$$
\Omega=\left\{(\mathbf{X}, E) \in \mathscr{C}^{(q)} \times \mathscr{E}: \sum_{\{x, y\} \in E} \sum_{s=1}^{q} 1_{X_{s}}(x)\left(1-1_{X_{s}}(y)\right)=0\right\} .
$$

Since the functions $(\mathbf{X}, x) \rightarrow 1_{X_{s}}(x)$ are measurable (see 5.1.9 in [24]) and $(\mathbf{X}, E) \rightarrow$ $\sum_{e \in E} g(\mathbf{X}, e)$ is measurable for any measurable $g$ on $\mathscr{C}^{(q)} \times E_{\mathbb{R}^{d}}$, this way of writing shows that $\Omega$ is measurable. We also have

$$
\mathbb{P}(\Omega) \geq P_{\Lambda \mid Y}^{z q}(\{Y\})=e^{-z q|\Lambda|} / Z_{\Lambda \mid Y}>0 .
$$

Thus we can introduce the conditional measure

$$
\mathbb{P}_{\Omega}=\mathbb{P}(\cdot \mid \Omega) .
$$

$\mathbb{P}_{\Omega}$ is the random-cluster representation measure with which the rest of this section is concerned.

Our first result is that if we disregard the edges, and look only at the particle positions and their types, then we obtain the continuum Potts model. We write pr for the projection from $\mathscr{C}^{(q)} \times \mathscr{E}$ onto $\mathscr{X}^{(q)}$.

Proposition 2.1. $\mathbb{P}_{\Omega} \circ \mathrm{pr}^{-1}=Q_{\Lambda \mid \mathbf{Y}}$.

Proof. For $\mathbf{X} \in \mathscr{X}_{\Lambda \mid \mathbf{Y}}^{(q)}$, let $\Omega_{\mathbf{X}}$ be the $\mathbf{X}$-section of $\Omega$. It follows straight from the definitions that, for $X=\operatorname{spX}$,

$$
\begin{aligned}
\mu_{X, \Lambda}\left(\Omega_{\mathbf{X}}\right) & =\prod_{e \in E_{X}: e \nsubseteq X_{s} \text { for any } s}\left(1-p_{\Lambda}(e)\right) \\
& =\exp \left[-H_{\mathbf{Y}}^{\varphi}(\mathbf{X})\right] .
\end{aligned}
$$


It is also easy to see that $\int \pi_{\Lambda}^{z q}(d X) \lambda_{X, \Lambda}$ is precisely the $q$-fold product measure $\left(\pi_{\Lambda}^{z}\right)^{q}$ on $\cdot X_{\Lambda}^{\cdot q}$ (which is in fact supported on $X_{\Lambda}^{\cdot q)}$ ). Hence, for any bounded measurable function $f$ on $\cdot X^{*}(q)$ we can write

$$
\begin{aligned}
\int f \circ \operatorname{pr} d \mathbb{P}_{\Omega} & =c_{1} \int_{\Omega} f \circ \operatorname{pr} d \mathbb{P} \\
& =c_{1} \int P_{\Lambda \mid Y}^{z q}(d X) \int \lambda_{X, \Lambda}(d \mathbf{X}) f(\mathbf{X}) \mu_{X, \Lambda}\left(\Omega_{\mathbf{X}}\right) \\
& =c_{2} \int\left(\pi_{\Lambda}\right)^{q}(d \mathbf{X}) f(\mathbf{X} \cup \mathbf{Y}) \exp \left[-H_{\Lambda \mid Y}^{\psi}(X)-H_{\Lambda \mid \mathbf{Y}}^{\varphi}(\mathbf{X})\right] \\
& =c_{3} \int f d Q_{\Lambda \mid \mathbf{Y}} .
\end{aligned}
$$

where $c_{1}, c_{2}, c_{3}$ are suitable constants. Since both $\mathbb{P}_{\Omega}$ and $Q_{\Lambda \mid \mathbf{Y}}$ are probability measures, we have $c_{3}=1$ and the proposition follows

Proposition 21 shows in particular that, under $\mathbb{P}_{\Omega}$, the conditional distribution of $\sigma(\mathbf{X})$ under the condition $\mathrm{sp} \mathbf{X}=X$ is nothing other than the Gibbs distribution for a discrete Potts model on the finite set $X$ with constant boundary condition 1 on $Y=X \backslash \Lambda$ It is therefore no surprise that the conditional distribution of $E$ under $\operatorname{sp} \mathbf{X}=X$ corresponds to the classical Fortuin-Kasteleyn random-cluster representation of the Potts model, as we will show next

Consider the mapping sp. $(\mathbf{X}, E) \rightarrow(\operatorname{spX}, E)$ from $\cdot X^{*}(q) \times \&$ onto $\mathscr{X} ; \times \mathscr{C}$. For each $(X, E) \in \cdot \mathscr{Y} \times \mathscr{\zeta}$ with $E \subseteq E_{X}$ we let $K(X, E)$ denote the number of connected components in the graph $(X, E)$. We will show in Lemma 24 below that $K(\cdot$,$) is measurable. We define the continuum random-cluster distribution C_{\Lambda \mid Y}$ on . $x^{\circ} \times$ \& by

$$
C_{\Lambda \mid Y}(d X, d E)=Z_{\Lambda \mid Y}^{-1} q^{K(X, E)} P_{\Lambda \mid Y}^{z}(d X) \mu_{X, \Lambda}(d E)
$$

where $P_{\Lambda \mid Y}^{z}$ is as in (5) with activity $z$ rather than $z q$, and $Z_{\Lambda \mid Y}$ is again a normalizing constant (different from the one in (5)). Recall that, for any $X \in X_{i \mid Y}$, the edges drawn by $\mu_{X, \Lambda}$ include all edges in $E_{Y}$ This means that $C_{\Lambda \mid Y}$ is defined by means of the so-called wired boundary condition. Note further that the definition (6) makes sense also for non-integer $q>0$.

Proposition 2.2. $\mathbb{P}_{\Omega} \circ \mathrm{sp}^{-1}=C_{\Lambda \mid Y}$

Proof. For $(X, E) \in \cdot \mathscr{x} \times \mathscr{\mho}$ with $X \in \mathscr{X}_{\lambda \mid Y}$ and $E_{Y} \subseteq E \subseteq E_{X}$ let

$$
\Omega_{(X, E)}=\left\{\mathbf{X} \in \cdot \mathscr{X}^{\cdot(q)} \cdot \operatorname{sp} \mathbf{X}=X,(\mathbf{X}, E) \in \Omega\right\}
$$

The definition of $\lambda_{X, \Lambda}$ then implies that

$$
\lambda_{X, \Lambda}\left(\Omega_{(X, E)}\right)=q^{K_{1}(X, E)} / q^{\#(X \cap \Lambda)},
$$

where $K_{\Lambda}(X, E)$ is the number of connected components of $(X, E)$ that are completely contained in $\Lambda$. Indeed, under $\lambda_{X, \Lambda}$ all $q^{\#(X \cap \Lambda)}$ possible values of $\sigma(\mathbf{X})$ have equal probability. Getting an element of $\Omega_{(X, E)}$ requires consensus of $\sigma(\mathbf{X})$ on each connected component of $(X, E)$, and $\sigma(\mathbf{X})$ is equal to 1 on the unique component 
containing $Y$; this means that each of the $K_{\Lambda}(X, E)$ connected components of $(X, E)$ not containing $Y$ has a choice between $q$ different values.

Proceeding as in the proof of Proposition 2.1, we obtain for any measurable function $f$ on $\mathscr{C}_{\Lambda} \times \mathscr{E}_{\Lambda \mid Y}$,

$$
\begin{aligned}
\int f \circ \operatorname{sp} d P_{\Omega} & =c_{1} \int P_{\Lambda \mid Y}^{z q}(d X) \int \mu_{X, \Lambda}(d E) f(X, E) \lambda_{X, \Lambda}\left(\Omega_{(X, E)}\right) \\
& =c_{2} \int P_{\Lambda \mid Y}^{z}(d X) \int \mu_{X, \Lambda}(d E) f(X, E) q^{K_{\Lambda}(X, E)}
\end{aligned}
$$

with suitable constants $c_{1}, c_{2}>0$. Here we used the fact that $P_{\Lambda \mid Y}^{z q}$ is absolutely continuous relative to $P_{\Lambda \mid Y}^{z}$ with density proportional to $X \rightarrow q^{\#(X \cap \Lambda)}$. Comparing this with the definition (6) of $C_{\Lambda \mid Y}$ we obtain the desired result because $K(X, E)-$ $K_{\Lambda}(X, E)$ is constant and equal to either 0 or 1 according to whether $Y=\emptyset$ or not.

Our third result in this section relates the influence of the boundary condition on the type of a single particle to connectivity probabilities in the random-cluster representation. For measurable $\Delta \subseteq \Lambda, 1 \leq s \leq q, \mathbf{X} \in \mathscr{X}^{(q)}$ and $(X, E) \in \mathscr{C} \times \mathscr{E}$ with $E \subseteq E_{X}$ we define

$$
N_{\Delta, s}(\mathbf{X})=\#\left(X_{s} \cap \Delta\right), \quad N_{\Delta}(\mathbf{X})=\sum_{s=1}^{q} N_{\Delta, s}(\mathbf{X})=\#(\operatorname{sp} \mathbf{X} \cap \Delta)
$$

and

$$
N_{\Delta \leftrightarrow \Lambda^{c}}(X, E)=\#\{x \in X \cap \Delta: \exists \text { a path in }(X, E) \text { from } x \text { to some } y \in X \backslash \Lambda\} .
$$

The functions $N_{\Delta, s}$ and $N_{\Delta}$ on $\mathscr{S}^{(q)}$ are measurable by definition, and the measurability of $N_{\Delta \leftrightarrow \Lambda^{c}}$ will be shown in Lemma 2.4 below.

Proposition 2.3. For any measurable $\Delta \subseteq \Lambda$,

$$
\int\left(q N_{\Delta, 1}-N_{\Delta}\right) d Q_{\Lambda \mid \mathbf{Y}}=(q-1) \int N_{\Delta \leftrightarrow \Lambda^{c}} d C_{\Lambda \mid Y}
$$

Proof. By Proposition 2.1, the integral on the left-hand side is equal to

$$
\mathbb{P}(\Omega)^{-1} \int P_{\Lambda \mid Y}^{z q}(d X) \int \mu_{X, \Lambda}(d E) \sum_{x \in X \cap \Delta} \int_{\Omega_{(X, E)}} \lambda_{X, \Lambda}(d \mathbf{X})\left(q 1_{\{\sigma(\mathbf{X}, x)=1\}}-1\right)
$$

where $\Omega_{(X, E)}$ is as in the proof of Proposition 2.2. If $x$ is connected to $\Lambda^{c}$ in $(X, E)$, the innermost integral is equal to $(q-1) \lambda_{X, \Lambda}\left(\Omega_{(X, E)}\right)$ because in this case the particle type $\sigma(x)=1$ on $\Omega_{(X, E)}$. Otherwise, $\sigma(x)$ is independent of $\Omega_{(X, E)}$ under $\lambda_{X, \Lambda}$ and takes all values in $\{1, \ldots, q\}$ with equal probability, which implies that the innermost integral vanishes. The result now follows as in the proof of Proposition 2.2.

We finally need to settle the measurability questions.

Lemma 2.4. (a) The mappings $X \rightarrow \lambda_{X, \Lambda}$ and $X \rightarrow \mu_{X, \Lambda}$ are probability kernels from $\mathscr{B}$ to $\mathscr{X}^{(q)}$ resp. $\mathscr{E}$.

(b) For any measurable $\Delta \subseteq \Lambda$, the functions $N_{\Delta \leftrightarrow \Lambda^{c}}$ and $K(\cdot, \cdot)$ on $\mathscr{B} \times \mathscr{E}$ are measurable. 
Proof (a) The fact that $\lambda_{X, \Lambda}$ depends measurably on $X$ is well-known in connection with the randomization of a point process; see [20], p. 17 and Lemma 1.7. As for $\mu_{X, \Lambda}$, we consider its Laplace transform $L_{X, \Lambda}$. For any measurable function $f$ : $E_{\text {足 } d} \rightarrow[0, \infty[$ we have

$$
\begin{aligned}
L_{X, \Lambda}(f) & :=\int \exp \left[-\sum_{e \in E} f(e)\right] \mu_{X, \Lambda}(d E) \\
& =\prod_{e \in E_{X}}\left[p_{\Lambda}(e) e^{-f(e)}+1-p_{\Lambda}(e)\right] \\
& =\exp \left[-\sum_{\{x, y\} \subseteq X} \tilde{f}(x, y)\right]
\end{aligned}
$$

where

$$
\tilde{f}(x, y)=-\log \left[e^{-f(\{x, y\})}+1_{\{\{x, y\} \cap \Lambda \neq \emptyset\}} e^{-\varphi(x-y)}\left(1-e^{-f(\{x, y\})}\right)\right] .
$$

Since $\tilde{f}$ is measurable, the function $X \rightarrow L_{X, \Lambda}(f)$ is measurable. Lemma 1.7 of [20] thus again implies that $\mu_{X, \Lambda}$ depends measurably on $X$.

(b) Consider the set $B$ of all quadrupels $(x, y, X, E) \in \mathbb{R}^{d} \times \mathbb{R}^{d} \times \mathscr{X}^{\cdot} \times \mathscr{\&}$ which are such that $x, y \in X, x \neq y$, and $x$ is connected to $y$ in the graph $\left(X, E \cap E_{X}\right)$. We may write $B=\bigcup_{n \geq 1} B_{n}$, where

$$
B_{1}=\{(x, y, X, E): x, y \in X,\{x, y\} \in E\}
$$

and, for $n \geq 1$,

$$
B_{n+1}=\left\{(x, y, X, E) \cdot \sum_{v \in X} 1_{B_{1}}(x, v, X, E) 1_{B_{n}}(v, y, X, E)>0\right\} .
$$

Since the functions $(x, X) \rightarrow 1_{X}(x),(x, y, E) \rightarrow 1_{E}(\{x, y\})$ and $X \rightarrow \sum_{v \in X} g(v, X)$ [for measurable $g$ ] are measurable (cf. 5.1.2 and 5.1.9 of [24]), it follows by induction that $B_{n}$ is measurable for any $n$, whence $B$ is also measurable.

Since

$$
N_{\Delta \leftrightarrow \Lambda^{c}}(X, E)=\sum_{x \in X \cap \Delta} g(x, X, E)
$$

with $g(x, X, E)=1$ when $\sum_{y \in X \backslash \Lambda} 1_{B}(x, y, X, E)>0$ and $g(x, X, E)=0$ otherwise, we now see that $N_{\Delta \leftrightarrow \Lambda^{c}}$ is measurable on $\mathscr{X}^{*} \times \mathscr{E}$. Also, for any $k \geq 1$ we have $K(X, E) \geq k$ if and only if

$$
\sum_{x_{1}, x_{k} \in X} \prod_{1 \leq \imath<j \leq k} 1_{\left\{x_{\imath} \neq x_{j}\right\}} 1_{B^{c}}\left(x_{\imath}, x_{\jmath}, X, E\right)>0
$$

In view of the above, the last expression depends measurably on $(X, E)$, and this yields the measurability of $K$. 


\section{Percolation in the Random-Cluster Model}

In this section we will establish the existence of percolation for continuum random cluster distributions $C_{\Lambda \mid Y}$ when $q \geq 1$ is an arbitrary real number, $z$ is sufficiently large and the boundary condition $Y$ contains sufficiently many particles.

For any box $\Delta \subset \mathbb{R}^{d}$ we set

$$
N_{\Delta \leftrightarrow \infty}(X, E)=\#\left\{x \in X \cap \Delta: x \text { belongs to an infinite cluster of }\left(X, E \cap E_{X}\right)\right\} \text {. }
$$

It follows from Lemma 2.4 that $N_{\Delta \leftrightarrow \infty}$ is measurable. We consider again the cells $\Delta(i)$ defined by (3) for the side length $\delta=r_{3} / \sqrt{d+3}$. The main result of this section is the following.

Proposition 3.1. Suppose assumptions (A1) to (A5) hold and $z$ is sufficiently large (depending on the parameters of our model). Then there exists an $\varepsilon>0$ and an integer $n_{*} \geq 1$ such that

$$
\int N_{\Delta \leftrightarrow \infty} d C_{\Lambda \mid Y} \geq \varepsilon
$$

for any cell $\Delta=\Delta(i)$, any finite union $\Lambda$ of cells, and any boundary condition $Y \in$ $\mathscr{C}_{\Lambda^{c}}$ with at least $n_{*}$ particles in each cell.

The proof of this proposition consists of two steps. First we will find a measure $\tilde{C}_{\Lambda \mid Y}$ which is stochastically smaller than $C_{\Lambda \mid Y}$ and such that, conditionally on the particle configuration, the edges are drawn independently of each other. It is therefore sufficient to establish percolation for $\tilde{C}_{\Lambda \mid Y}$. This will be done by discretization and comparison with a mixed site-bond percolation problem on the lattice $\mathbb{Z}^{d}$.

The definition of $\tilde{C}_{\Lambda \mid Y}$ is based on the following two ingredients.

- The distribution of particle positions is given by the marginal distribution

$$
M_{\Lambda \mid Y}=C_{\Lambda \mid Y}(\cdot \times \mathscr{E})
$$

of $C_{\Lambda \mid Y}$ on $\mathscr{X}$.

- For given $X \in \mathscr{B}$, the distribution $\tilde{\mu}_{X}$ of edges is defined as the distribution of the random set $\left\{e \in E_{X}: \tilde{\eta}_{e}=1\right\}$, where $\left(\tilde{\eta}_{e}\right)_{e \in E_{X}}$ are independent $\{0,1\}$-valued random variables with

$$
\operatorname{Prob}\left(\tilde{\eta}_{e}=1\right)=\tilde{p}:=\frac{1-e^{-u}}{q e^{-u}+1-e^{-u}} \text { when } e=\{x, y\} \text { with }|x-y| \leq r_{3}
$$

and $\operatorname{Prob}\left(\tilde{\eta}_{e}=1\right)=0$ otherwise; the constants $r_{3}$ and $u$ are as in assumption (A1). It follows as in Lemma 2.4 that $X \rightarrow \tilde{\mu}_{X}$ is a probability kernel from $\mathscr{C}$ to $\mathscr{E}$.

We now define $\tilde{C}_{\Lambda \mid Y}$ on $\mathscr{B} \times \mathscr{E}$ by

$$
\tilde{C}_{\Lambda \mid Y}(d X, d E)=M_{\Lambda \mid Y}(d X) \tilde{\mu}_{X}(d E) .
$$

For the sake of comparison we note from (6) that

$$
C_{\Lambda \mid Y}(d X, d E)=M_{\Lambda \mid Y}(d X) \mu_{X, \Lambda}^{q}(d E),
$$

where 


$$
\mu_{X, \Lambda}^{q}(d E)=q^{K(X, E)} \mu_{X, \Lambda}(d E) / \int q^{K(X,)} d \mu_{X, \Lambda}
$$

is the discrete random-cluster distribution on $X \cap A$ with wired boundary condition on the set $X \backslash A$

To state the relationship between $C_{\Lambda \mid Y}$ and $\tilde{C}_{\Lambda \mid Y}$ we recall that a probability measure $\nu_{1}$ on a partially ordered measurable space is said to dominate a second probability measure $\nu_{2}$ if $\int f d \nu_{1} \geq \int f d \nu_{2}$ for any increasing bounded measurable function $f$ In this case we write $\nu_{1} \succeq \nu_{2}$

Lemma 3.2. For all $q \geq 1$ and $X \in \mathcal{X} ;$, we have $\mu_{X . \Lambda}^{q} \succeq \tilde{\mu}_{X}$ and therefore $C_{\Lambda \mid Y} \succeq$ $\tilde{C}_{\Lambda \mid Y}$.

Proof By assumption (A1),

$$
p_{\Lambda}(e) / q\left(1-p_{\Lambda}(e)\right) \geq \tilde{p} /(1-\tilde{p})
$$

for all $e \in E$ The relation $\mu_{X . \Lambda}^{q} \succeq \tilde{\mu}_{X}$ thus follows from a well-known comparison inequality for discrete random-cluster distributions; see e.g. inequality (7.11) of [16] (this reference deals only with the case of equal edge probabilities, but the proof works without change in the general case). The second assertion is immediate from (8) and (9)

Since $N_{\Delta \leftrightarrow \infty}$ is increasing, Lemma 3.2 shows that in order to prove Proposition 31 it is sufficient to prove the analogous result for $\tilde{C}_{\Lambda \mid Y}$. In the case $\psi \equiv 0$, this can be achieved by a further stochastic comparison. Namely, the point-process analog of Holley's inequality $[29,12]$ together with Lemma 3.5 below then imply that $M_{\Lambda \mid Y} \succeq$ $\pi_{A}^{\alpha z}$, so that Proposition 31 follows from the fact that the so-called random connection model of Poisson particles with random edges exhibits percolation when the particle density is large enough $[28,25]$. In the general case such a stochastic comparison does not work. Nevertheless, it is possible to extend the proof of percolation in the random connection model to our present setting. Specifically, we shall use a discretization to compare the percolation problem for $\tilde{C}_{\Lambda \mid Y}$ with a site-bond percolation problem on the lattice $\mathbb{Z}^{d}$.

The basic idea is as follows We consider the division of $\mathbb{R}^{d}$ into the cells $\Delta(i)$ defined by (3). The side length $\delta$ is chosen so small that any two points in adjacent cells have distance less than $r_{3}$, which means that $\delta=r_{3} / \sqrt{d+3}$. For a suitably chosen number $n_{*}$, we will call a cell good relative to a given particle-edge configuration if there exist at least $n_{*}$ particles in the cell and these particles, together with the associated edges, form a connecte $d$ graph. Two neigboring cells will be said to be linked if there exists an edge joining a particle in one cell to a particle in the other cell. An infinite cluster of particles joined by edges then certainly exists whenever there exists an infinite cluster of good cells joined by links This is a correlated mixed site-bond percolation problem on $\mathbb{Z}^{d}$ For suitably chosen parameters, percolation in this lattice model can be established by means of a comparison with the Bernoulli site-bond percolati on problem to which we turn now

Consider the integer lattice $\mathbb{Z}^{d}$, and let $0 \leq p \leq 1$ Declare a site $i \in \mathbb{Z}^{d}$, or a bond $\{i, j\}$ between two sites of distance 1 , to be open with probability $p$, and closed otherwise These designations are done independently for all sites and bonds Let $\theta(p)$ denote the probability that the origin is open and belongs to an infinite component of the open graph consisting of open sites and open bonds. We shall use the following fact. 
Lemma 3.3. For $d \geq 2$, there exists a threshold value $p_{c}<1$ (depending on $d$ ) such that $\theta(p)>0$ when $p>p_{c}$.

Proof. This follows either by an adaptation of the usual Peierls argument, or by an application of an inequality of McDiarmid and Hammersley (see inequality (10.8) in [21]) which implies that $\theta(p)$ exceeds the corresponding probability for the Bernoulli pure site percolation problem with parameter $p^{2}$.

We shall also take advantage of the following well-known fact on random graphs.

Lemma 3.4. For any positive integer $n$ and $0<p<1$, let $\mathscr{G}_{n, p}$ be the random graph on $n$ vertices where each pair of vertices independently forms an edge with probability $p$. Then, for fixed $p$,

$$
\gamma(n, p)=\operatorname{Prob}\left(\mathscr{G}_{n, p} \text { is connected }\right) \rightarrow 1 \text { as } n \rightarrow \infty .
$$

In particular, $\gamma(p)=\inf _{n \geq 1} \gamma(n, p)>0$.

Proof. The first statement follows from the simple observation that $\mathscr{G}_{n, p}$ is certainly connected if the first vertex is connected to any other vertex by a path of length 2. A straightforward estimate shows that the latter event has probability at least $1-(n-1)\left(1-p^{2}\right)^{n-2}$. This also gives the second assertion because each $\gamma(n, p)$ is positive. (A complete account of the asymptotic behaviour of $\mathscr{G}_{n, p}$ can be found in [1].)

We finally need a third preparatory lemma which will allow us to control the measure $M_{\Lambda \mid Y}$. For $X \in \mathscr{B}_{\Lambda \mid Y}$ let

$$
h_{\Lambda}(X)=Z_{\Lambda \mid Y}^{-1} \int q^{K(X,)} d \mu_{X, \Lambda},
$$

where $Z_{\Lambda \mid Y}$ is as in (6). Note that $h_{\Lambda}$ is the Radon-Nikodym derivative of $M_{\Lambda \mid Y}$ with respect to $P_{\Lambda \mid Y}^{z}$.

Lemma 3.5. For $q \geq 1$, there exists a constant $\alpha>0$ (depending on $q, r_{4} / r_{3}$ and $\tilde{p}$ ) such that for any box $\Lambda \subset \mathbb{R}^{d}, X \in \mathscr{C}_{\Lambda \mid Y}$ and $x \in \Lambda \backslash X$,

$$
h_{\Lambda}(X \cup\{x\}) \geq \alpha h_{\Lambda}(X) .
$$

Proof. Since $x \in \Lambda$, we can ignore the normalizing factor $Z_{\Lambda \mid Y}$. Let $\mu_{x \mid X, \Lambda}$ denote the distribution of the random edge set $\left\{e \in E_{X \cup x} \backslash E_{X}: \eta_{e}=1\right\} \in \mathscr{E}$, where $\left(\eta_{e}\right)$ are independent $\{0,1\}$-valued with $\operatorname{Prob}\left(\eta_{e}=1\right)=p_{\Lambda}(e)$. (We omit the braces around $x$ for brevity.) Then we can write

$$
h_{\Lambda}(X \cup x) / h_{\Lambda}(X)=\int \mu_{X, \Lambda}^{q}(d E) \int \mu_{x \mid X, \Lambda}\left(d E^{\prime}\right) q^{K\left(X \cup x, E \cup E^{\prime}\right)-K(X, E)}
$$

with $\mu_{X, \Lambda}^{q}$ as in (9). To estimate the last expression we note that, due to the finite range assumption (A2), all particles of $X$ joined to $x$ by $\mu_{x \mid X, \Lambda}$ belong to the ball $B=\left\{y \in \mathbb{R}^{d}:|x-y| \leq r_{4}\right\}$. We split $B$ into a minimal number $k$ of disjoint Borel sets $B_{1}, \ldots, B_{k}$ of diameter at most $r_{3} ; k$ obviously depends only on the ratio $r_{4} / r_{3}$. Writing $X^{j}=X \cap B_{j}$ for the configuration in $B_{j}$, we let $A_{j}$ denote the set of all $E \in \mathscr{E}$ for which the graph $\left(X^{j}, E \cap E_{X^{j}}\right)$ is connected, $1 \leq j \leq k$. For 
$E \in A=\bigcap_{j=1}^{k} A_{j}$, there exist at most $k$ different clusters of $(X, E)$ which can be joined together by the edges in $E^{\prime}$ drawn by $\mu_{x \mid X, \Lambda}$. Hence $K\left(X \cup x, E \cup E^{\prime}\right) \geq$ $K(X, E)-k$ when $E \in A$. This gives us the first inequality in

$$
\begin{aligned}
h_{\Lambda}(X \cup x) / h_{\Lambda}(X) & \geq q^{-k} \mu_{X, \Lambda}^{q}(A) \\
& \geq q^{-k} \tilde{\mu}_{X}(A) \\
& \geq q^{-k} \gamma(\tilde{p})^{k}=\cdot \alpha
\end{aligned}
$$

The second inequality above comes from Lemma 3.2 because $A$ is increasing. The last estimate follows from the fact that $A_{1}, \quad, A_{k}$ are independent under $\tilde{\mu}_{X}$, each $A_{j}$ having probability at least $\gamma(\tilde{p})$ by Lemma 3.4

We now turn to the comparison of the percolation problem for $\tilde{C}_{\Lambda \mid Y}$ with the Bernoulli site-bond percolation problem on $\mathbb{Z}_{s}^{d}$ First of all, we introduce the number $n_{*}$ on which the definition of good cells is based. To this end we fix any $p_{*}$ with $p_{c}<p_{*}<1$, where $p_{c}$ is as in Lemma 3.3. By Lemma 3.4, we can find a number $n_{*}$ such that

$$
\gamma(n, \tilde{p}) \geq \sqrt{p_{*}} \text { and } \lambda(n, \tilde{p})=1-(1-\tilde{p})^{n^{2}} \geq p_{*} \quad \text { when } n \geq n_{*}
$$

Note that $\lambda\left(n_{*}, \tilde{p}\right)$ is a lower bound of the $\tilde{\mu}_{X}$-probability for the the existence of at least one edge between particles in neighboring cells when each cell contains at least $n_{*}$ particles.

Having defined $n_{*}$, we are in a position to specify the second scale condition in assumption (A5) We require that $r_{1}$ is so small that

$$
\left(n_{*}-1\right) v\left(r_{1}\right)<\left(\delta-2 r_{2}\right)^{d},
$$

where $v\left(r_{1}\right)$ is the volume of a ball with radius $r_{1}$. Since $n_{*}$ depends on $\tilde{p}$ (and $d$ ) by (10), this condition on $r_{1}$ involves $u$ and $q$ in addition to $r_{2}$ and $r_{3}$. In particular, if $u$ is so large that we can choose $n_{*}=1$ then condition (11) becomes trivial, in which case we can take $r_{1}=r_{2}$.

The key step in the proof of Proposition 3.1 is to estimate the conditional probability that a cell $\Delta(i)$ contains at least $n_{*}$ particles, given the configuration outside of $\Delta(i)$. For arbitrary $i \in \mathbb{Z}^{d}$, we use again the abbreviation $N_{\imath}=N_{\Delta(\imath)}$ for the counting variable associated to $\Delta(i)$, and for any box $\Lambda \supset \Delta(i)$ and any $Y^{\prime} \in \mathscr{X}^{\circ} \Delta(i)^{c}$ with $Y^{\prime} \backslash \Lambda=Y$ we consider the conditional distribution $M_{\Lambda, \imath \mid Y^{\prime}}$ of the particle configuration in $\Delta(i)$ given the configuration $Y^{\prime}$ in $\Delta(i)^{c}$ relative to $M_{\Lambda \mid Y}$

Lemma 3.6. If $r_{1}, r_{2}, r_{3}$ satisfy (A5) as specified by (11) and $z$ is sufficiently large, we have

$$
M_{\Lambda, i \mid Y^{\prime}}\left(N_{\imath} \geq n_{*}\right) \geq \sqrt{p_{*}}
$$

for all $i \in \mathbb{R}$, all $\Lambda \subset \mathbb{R}^{d}$ which are a finite union of cells, each tempered $Y \in \cdot X_{\Lambda^{c}}$ with $N_{j}(Y) \geq n_{*}$ for all cells $\Delta(j) \subset \Lambda^{c}$, and any $Y^{\prime} \in \mathscr{X}_{\Delta(i)^{\mathrm{c}}}$ with $Y^{\prime} \backslash \Lambda=Y$.

Proof. We can assume that $\Delta=\Delta(i)$ is contained in $A$ because otherwise the assertion holds trivially Then

$$
M_{\Lambda, \imath \mid Y^{\prime}}(d X)=Z_{\Lambda, \imath \mid Y^{\prime}}^{-1} h_{\Lambda}\left(X \cup Y^{\prime}\right) \exp \left[-H_{\Delta \mid Y^{\prime}}^{\psi}(X)\right] \pi_{\Delta}(d X)
$$


$X \in \mathscr{C}_{\Delta}$, with a normalizing constant $Z_{\Lambda, i \mid Y^{\prime}}$. By (2), this implies that for any $n \geq 0$,

$$
\frac{M_{\Lambda, i \mid Y^{\prime}}\left(N_{i}=n+1\right)}{M_{\Lambda, i \mid Y^{\prime}}\left(N_{i}=n\right)}=\frac{z}{n+1} \int M_{\Lambda, i \mid Y^{\prime}}\left(d X \mid N_{i}=n\right) g_{i \mid Y^{\prime}}(X)
$$

with

$$
g_{i \mid Y^{\prime}}(X)=\int_{\Delta} d x \exp \left[-\sum_{y \in X \cup Y^{\prime}} \psi(x-y)\right] h_{\Lambda}\left(X \cup Y^{\prime} \cup x\right) / h_{\Lambda}\left(X \cup Y^{\prime}\right) .
$$

By Lemma 3.5, the last ratio of $h_{\Lambda}$ 's is at least $\alpha$, so that we only need to look at the exponential term. Consider the reduced cell $\Delta_{0}$ which is obtained from $\Delta$ by removing a boundary layer of thickness $r_{2}$. By (A5), the volume $\left(\delta-2 r_{2}\right)^{d}$ of $\Delta_{0}$ is still positive. Together with (A4), this gives us the lower bound

$$
g_{i \mid Y^{\prime}}(X) \geq \alpha \int_{\Delta_{0}} d x \exp \left[-\sum_{y \in X} \psi(x-y)\right]
$$

which is independent of $Y^{\prime}$.

To estimate this further we use an argument of Dobrushin and Minlos [6]. Consider the set

$$
\Delta_{X}=\left\{x \in \Delta_{0}:|x-y| \geq r_{1} \text { for all } x \in X\right\} .
$$

If $N_{i}(X)=n<n_{*}$ then

$$
\left|\Delta_{X}\right| \geq\left|\Delta_{0}\right|-\left(n_{*}-1\right) v\left(r_{1}\right)=: v_{*}>0
$$

by assumption (11). Here $|\cdot|$ stands for the volume of a set in $\mathbb{R}^{d}$. On the other hand, Markov's inequality yields for any $c>0$,

$$
\left|\left\{x \in \Delta_{X}: \sum_{y \in X} \psi(x-y) \geq c\right\}\right| \leq\left(n_{*}-1\right) b\left(r_{1}\right) / c
$$

where $b\left(r_{1}\right)$ is the integral in assumption (A4). This shows that

$$
g_{i \mid Y^{\prime}}(X) \geq \alpha e^{-c}\left[v_{*}-\left(n_{*}-1\right) b\left(r_{1}\right) / c\right]
$$

when $N_{i}(X)<n_{*}$. Choosing $c$ sufficiently large, we find that there exists a constant $b>0$ such that $g_{i \mid Y^{\prime}}(X) \geq b n_{*}$ whenever $N_{i}(X)<n_{*}$. We thus arrive at the conclusion that the ratio in (12) is at least $b z$ when $n<n_{*}$. Hence

$$
\begin{aligned}
M_{\Lambda, i \mid Y^{\prime}}\left(N_{i}<n_{*}\right) & \leq \sum_{n=0}^{n_{*}-1} M_{\Lambda, i \mid Y^{\prime}}\left(N_{i}=n_{*}\right)\left(\frac{1}{b z}\right)^{n_{*}-n} \\
& \leq(b z-1)^{-1}
\end{aligned}
$$

when $b z>1$. The lemma thus follows when $z$ is so large that $1-(b z-1)^{-1} \geq \sqrt{p_{*}}$. 
We now return to the picture of good cells and links between good cells. Suppose $z$ is as large as required by Lemma 3.6, and $\Lambda$ is a finite union of cells. By (10) and the choice of $z$, the conditional probability under $\tilde{C}_{\Lambda \mid Y}$ of the event that a cell $\Delta(i)$ is good given the particle configuration $Y^{\prime}$ outside of $\Delta(i)$ and all edges which are not contained in $\Delta(i)$ is at least

$$
\int_{\left\{N_{\imath} \geq n_{*}\right\}} M_{\Lambda, \imath \mid Y^{\prime}}(d X) \gamma\left(N_{\imath}(X), \tilde{p}\right) \geq p_{*}
$$

Condition (10) also guarantees that the conditional probability for two neighboring cells to be linked when they are good is not less than $\lambda\left(n_{*}, \tilde{p}\right) \geq p_{*}$. By a standard comparison argument, we can conclude that

$$
\int N_{\Delta(\imath) \leftrightarrow \infty} d \tilde{C}_{\Lambda \mid Y} \geq n_{*} \theta\left(p_{*}\right)=: \varepsilon>0
$$

for such $z$ and $\Lambda$ and all $i$. Together with Lemma 3.2, this observation completes the proof of Proposition 3.1.

We now specialize to the case $q=1$. Let $P$ be a tempered Gibbs measure on. $\mathscr{X}$. with pair potential $\psi$ and activity $z$. In complete analogy to Sect. 1 , this means that $P$ is supported on the tempered configurations in . $X^{*}$ and, for any finite box $\Lambda$ in $\mathbb{R}^{d}$, admits $P_{\Lambda \mid Y}^{z}$ as conditional distribution under the condition $X \backslash \Lambda=Y$ Assumptions (A3) and (A4) imply that such a $P$ exists; see Remark 42 By the case $q=1$ of Lemma 3.6, the conditional probabilities of $P$ satisfy $P_{\Delta(\imath) \mid Y^{\prime}}^{z}\left(N_{\imath} \geq n_{*}\right) \geq \sqrt{p_{*}}$ for all $i \in \mathbb{Z}^{d}$ and all tempered $Y^{\prime} \in \mathscr{X}_{i(2)^{c}}$ when $z$ is large enough. The argument above thus gives the following corollary of independent interest

Corollary 3.7. Suppose $X$ is a configuration of particles chosen at random according to a tempered Gibbs measure $P$ with pair interaction $\psi$ and activity z. Suppose further that any two distinct particles $x, y \in X$ with $|x-y| \leq r_{3}$ are connected by an edge with probability $\tilde{p}>0$, independently for all such pairs of particles. If (A3) to (A5) hold and $z$ is sufficiently large then, with probability 1 , there exists an infinite cluster of particles connected by edges. Explicitly, the measure $\tilde{C}_{P}(d X, d Y)=P(d X) \tilde{\mu}_{X}(d E)$ satisfies

$$
\tilde{C}_{P}\left(\sup _{\imath \in \mathbb{R}^{d}} N_{\Delta(\imath) \leftrightarrow \infty} \geq 1\right)=1
$$

In the case of no interaction $(\psi \equiv 0)$ when $P$ is a Poisson point process, this result was obtained first by $M$. Penrose [28] (It is then not necessary to assume that the edge probabilities are uniformly positive for short distances.)

\section{The Infinite-Volume Limit}

In this section we will complete the proof of Theorem 1.1 by investigating the Gibbs distributions $Q_{\Lambda \mid \mathbf{Y}}$ in the infinite volume limit $\Lambda \uparrow \mathbb{R}^{d}$. Many of our arguments are standard, but we have to be careful with various details

We assume again that $q$ is an integer $\geq 2$. As before, we consider the cells $\Delta(i)$ defined by (3) with $\delta=r_{3} / \sqrt{d+3}$. For notational convenience we assume without loss of generality that the units in $\mathbb{R}^{d}$ are chosen in such a way that $\delta=1$. The limit $\Lambda \uparrow \mathbb{E}^{d}$ will be taken along the sequence 


$$
\Lambda(n)=\left[-n-1 / 2, n+1 / 2\left[^{d}\right.\right.
$$

of boxes of volume $v_{n}=(2 n+1)^{d}$.

Let $Y \in \mathscr{X}$ be any configuaration with a fixed number $n_{*}$ of particles in each cell, i.e., $N_{i}(Y)=n_{*}$ for all $i \in \mathbb{Z}^{d}$. For each $n, Y$ defines the boundary condition

$$
\mathbf{Y}(n)=(Y \backslash \Lambda(n), \emptyset, \ldots, \emptyset) \in \mathscr{X}_{\Lambda(n)^{c}}^{(q)}
$$

of particles of type 1 . We look at the projection

$$
Q_{n}=Q_{\Lambda(n) \mid \mathbf{Y}(n)}\left(\mathbf{X} \in \mathscr{C}^{(q)}: \mathbf{X} \cap \Lambda(n) \in \cdot\right)
$$

of $Q_{\Lambda(n) \mid \mathbf{Y}(n)}$ to $\Lambda(n)$; here $\mathbf{X} \cap \Lambda(n)=\left(X_{s} \cap \Lambda(n)\right)_{1 \leq s \leq q} \in \mathscr{K}_{\Lambda(n)}^{(q)}$ is the restriction of $\mathbf{X} \in \mathscr{C}^{(q)}$ to $\Lambda(n)$.

To obtain asymptotic translation invariance we take a spatial average of $Q_{n}$. For $x \in \mathbb{R}^{d}$, the translation $\vartheta_{x}: \mathscr{K}^{(q)} \rightarrow \mathscr{C}^{(q)}$ is defined by

$$
\vartheta_{x} \mathbf{X}=\left(\left\{y-x: y \in X_{s}\right\}\right)_{1 \leq s \leq q}
$$

It is well-known [24] that the mapping $(x, \mathbf{X}) \rightarrow \vartheta_{x} \mathbf{X}$ is measurable. In view of the cell structure we have imposed on $\mathbb{R}^{d}$, we will confine ourselves first to lattice translations. So we define

$$
\bar{Q}_{n}=v_{n}^{-1} \sum_{i \in L(n)} Q_{n} \circ \vartheta_{i}^{-1}
$$

with $L(n)=\Lambda(n) \cap \mathbb{Z}^{d}$. We will show that the sequence $\left(\bar{Q}_{n}\right)$ admits an accumulation point in a fairly strong topology which is defined as follows.

Let $\mathscr{L}$ denote the class of all measurable functions $f: \mathscr{C}^{(q)} \rightarrow \mathbb{R}$ which are local and tame, in that there exists some $\ell \geq 1$ such that $f=f(\cdot \cap \Lambda(\ell))$ and $|f| \leq b\left(1+N_{\Lambda(\ell)}\right)$ for some constant $b=b(f)<\infty$. The topology $\tau_{\mathscr{C}}$ of local convergence on the set of all probability measures $R$ on $\mathscr{X}^{(q)}$ with $\int N_{\Delta} d R<\infty$ for each finite box $\Delta$ is then defined as the weak* topology induced by $\mathscr{B}$, i.e., as the smallest topology for which the mappings $R \rightarrow \int f d R$ with $f \in \mathscr{B}$ are continuous.

The basic result of this section is the following.

Proposition 4.1. Suppose $\varphi$ and $\psi$ satisfy (A1) to (A4) and $z>0$ is arbitrary. Then there exists a subsequence of $\left(\bar{Q}_{n}\right)$ which converges in $\tau_{\mathscr{L}}$ to a limit $Q$. This $Q$ is a continuum Potts measure for $\varphi, \psi$ and $z$. It is invariant under the lattice translations $\left(\vartheta_{i}\right)_{i \in \mathbb{Z}^{d}}$ and of second order in the sense that $\int N_{\Delta}^{2} d Q<\infty$ for each finite box $\Delta \subset \mathbb{R}^{d}$.

Let us show first how Theorem 1.1 follows from the preceding proposition and the results of the foregoing sections. Suppose $z$ and $n_{*}$ are as large as required by Proposition 3.1. Let $Y$ be a configuration with $n_{*}$ particles in each cell and an associated limiting measure $Q$ be chosen according to Proposition 4.1. To achieve invariance under the full translation group $\left(\vartheta_{x}\right)_{x \in \mathbb{R}^{d}}$ we pass from $Q$ to the average

$$
Q^{(1)}=\int_{\Delta(0)} Q \circ \vartheta_{x}^{-1} d x
$$


$Q^{(1)}$ is a continuum Potts measure of second order Also, $Q^{(1)}$ is symmetric under exchanges of the types $2, \ldots, q$ because all $Q_{n}$ exhibit this symmetry.

Applying Propositions 2.3 and 31 we see that, for $\Delta=\Delta(0)$,

$$
\begin{aligned}
\int\left(q N_{\Delta, 1}-N_{\Delta}\right) d \bar{Q}_{n} & \geq(q-1) v_{n}^{-1} \sum_{i \in L(n)} \int N_{\Delta(i) \leftrightarrow \infty} d C_{\Lambda(n) \mid Y \backslash \Lambda(n)} \\
& \geq(q-1) \varepsilon,
\end{aligned}
$$

and also that the expression on the left-hand side is increasing in $\Delta$. Since $N_{\Delta, 1}, N_{\Delta} \in$ $\%$, the same inequality holds for $Q$ in place of $\bar{Q}_{n}$, and using the monotonicity in $\Delta$ we obtain that

$$
\int\left(q N_{\Delta, 1}-N_{\Delta}\right) d Q^{(1)}>0
$$

for $\Delta=\left[-1,1\left[^{d}\right.\right.$, and by translation invariance also for $\Delta=\Delta(0)$. Together with the symmetry of $Q^{(1)}$, this means that

$$
\rho_{1}\left(Q^{(1)}\right)>\rho_{2}\left(Q^{(1)}\right)=\quad=\rho_{q}\left(Q^{(1)}\right),
$$

where $\rho_{s}\left(Q^{(1)}\right)=\int N_{\Delta(0), s} d Q^{(1)}$ is the expected density of particles of type $s$. For

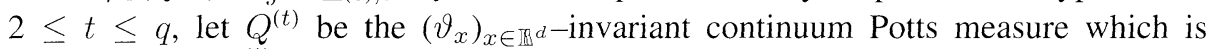
obtained from $Q^{(1)}$ by interchanging the roles of the types 1 and $t$. It is then obvious that the matrix $\left(\rho_{s}\left(Q^{(t)}\right)_{1 \leq s, t \leq q}\right.$ is regular. By the ergodic decomposition theorem (which can be derived in analogy to the lattice case considered in Chapter 14 of [9]), this implies the existence of $\left(\vartheta_{x}\right)_{x \in \mathbb{R}^{d}}$-ergodic continuum Potts measures with the same property; cf. Corollary (14.18) and Comment (16.19) in [9] The proof of Theorem 11 is thus complete To conclude, let us note that our estimates also imply that $\rho_{s}\left(Q^{(s)}\right) / \rho\left(Q^{(s)}\right) \rightarrow 1$ as $z \rightarrow \infty$ for each type $s$.

We now turn to the proof of Proposition 4.1. We might follow the lines of Ruelle [30] or, under slightly stronger assumptions on $\psi$, of Dobrushin [4, 5]; these references, however, work with other topologies than $\tau_{y}$. We prefer to sketch here an alternative "thermodynamic" approach in the spirit of large deviation theory. We proceed in four stages.

Step 1. Energy and entropy estimates. Suppose $\psi$ is superstable and lower regular. By definition, superstability means that there exist constants $a, b>0$ such that, for all $n$ and $X \in \cdot x_{\Lambda(n)}$,

$$
H^{\psi}(X) \geq \sum_{\imath \in L(n)}\left[a N_{\imath}(X)^{2}-b N_{\imath}(X)\right]
$$

Lower regularity is defined as the existence of numbers $\Psi_{i} \geq 0, i \in \mathbb{Z}^{d}$, such that $\Psi:=\sum_{\imath \in \mathbb{Z}^{d}} \Psi_{i}<\infty$ and

$$
\sum_{x \in X \cap \Delta(i), y \in X \cap \Delta(j)} \psi(x-y) \geq-\Psi_{\imath-\jmath} N_{\imath}(X) N_{\jmath}(X)
$$

for all $X \in . \not$; and $i, j \in \mathbb{Z}$. For our fixed configuration $Y$, both conditions together imply that

$$
H_{\Lambda(n) \mid \mathbf{Y}(n)} \geq \sum_{\imath \in L(n)}\left[a N_{i}^{2}-b_{*} N_{\imath}\right] \geq-c_{*} v_{n}
$$

for all $n$, where $b_{*}=b+\Psi n_{*}$ and $c_{*}=b_{*}^{2} / 4 a$. 
As is well-known, the relative entropy (or Kullback-Leibler information) of two probability measures $\mu, \nu$ on the same measurable space is defined by $I(\mu ; \nu)=$ $\int \log f d \mu \geq 0$ if $\mu$ has Radon-Nikodym density $f$ relative to $\nu$, and $I(\mu ; \nu)=\infty$ otherwise. Using (1) we obtain for each $n$, writing $\pi_{n, q}=\left(\pi_{\Lambda(n)}\right)^{q}$,

$$
\begin{aligned}
v_{n}^{-1} I\left(Q_{n} ; \pi_{n, q}\right) & =-v_{n}^{-1} \int H_{\Lambda(n) \mid \mathbf{Y}(n)} d Q_{n}-v_{n}^{-1} \log Z_{\Lambda(n) \mid \mathbf{Y}(n)} \\
& \leq c_{*}+z q=: c .
\end{aligned}
$$

The inequality follows from the energy estimate (15) and the fact that $Z_{\Lambda(n) \mid \mathbf{Y}(n)} \geq$ $\pi_{\Lambda(n)}(\{\emptyset\})^{q}$. In the case $\psi \geq 0$, the preceding inequality (16) holds trivially with $c=z q$.

Step 2. The existence of an accumulation point $Q$. We first need to introduce the relative entropy density. For any $\left(\vartheta_{i}\right)_{i \in \mathbb{Z}^{d}}$-invariant probability measure $R$ on $\mathscr{C}^{(q)}$, this is defined as

$$
I(R)=\lim _{k \rightarrow \infty} v_{k}^{-1} I\left(R_{(k)} ; \pi_{k, q}\right)
$$

where $R_{(k)}$ is the projection of $R$ onto $\mathscr{C}_{\Lambda(k)}^{(q)}$. For the existence of this limit we refer to [9], Theorem 15.12. The important fact we need here is that the sublevel sets $\{I \leq c\}$ are sequentially compact in the topology $\tau_{\mathscr{B}}$. This was shown in Proposition 2.6 of [13].

We now consider the following particular sequence $\left(\tilde{Q}_{n}\right)$ of $\left(\vartheta_{i}\right)_{i \in \mathbb{Z}^{d}}$-invariant measures. For each $n$, we write $\hat{Q}_{n}$ for the probability measure on $\mathscr{X}^{(q)}$ relative to which the particle configurations in the disjoint blocks $\Lambda(n)+(2 n+1) i, i \in \mathbb{Z}^{d}$, are independent with identical distribution $Q_{n}$, and we set

$$
\tilde{Q}_{n}=v_{n}^{-1} \sum_{\imath \in L(n)} \hat{Q}_{n} \circ \vartheta_{i}^{-1}
$$

It is not difficult to see that $I\left(\tilde{Q}_{n}\right)=v_{n}^{-1} I\left(Q_{n} ; \pi_{n, q}\right)$, cf. the proof of Proposition (16.34) in [9]. Together with (16) this shows that the sequence $\left(\tilde{Q}_{n}\right)$ has a subsequential limit $Q$ in $\tau_{\mathscr{C}}$. Clearly, $Q$ inherits the $\left(\vartheta_{i}\right)_{i \in \mathbb{Z}^{d}}$-invariance of the $\tilde{Q}_{n}$. Now the point is that, by a slight variant of Lemma 5.7 in [13], the sequences $\left(\tilde{Q}_{n}\right)$ and $\left(\bar{Q}_{n}\right)$ are asymptotically equivalent in $\tau_{\mathscr{C}}$. We thus conclude that a subsequence of $\left(\bar{Q}_{n}\right)$ converges to $Q$.

Step 3. Bounds on the second moments. Suppose first that $\psi$ is superstable and lower regular, and let $i \in \mathbb{Z}^{d}$ and $n \geq 0$ be arbitrary. Using the estimates (15) and (16) together with the nonnegativity of the relative entropy we find

$$
\begin{aligned}
\int N_{i}^{2} d \bar{Q}_{n} & =v_{n}^{-1} \sum_{\jmath \in L(n)} \int N_{\imath+\jmath}^{2} d Q_{n} \\
& \leq n_{*}^{2}+v_{n}^{-1} \sum_{\jmath \in L(n)} \int N_{j}^{2} d Q_{n} \\
& \leq n_{*}^{2}+\frac{2}{a v_{n}} \int H_{\Lambda(n) \mid \mathbf{Y}(n)} d Q_{n}+\int\left(\frac{2 b_{*}}{a} N_{0}-N_{0}^{2}\right) d \bar{Q}_{n} \\
& \leq n_{*}^{2}+2 z q / a+\left(b_{*} / a\right)^{2}=: m .
\end{aligned}
$$

In the alternative case when $\psi \geq 0$, a uniform bound on $\int N_{i}^{2} d \bar{Q}_{n}$ can be obtained from the fact that, in this case, the density of $Q_{\Delta(i) \mid \mathbf{X}}$ relative to $\left(\pi_{\Delta(i)}\right)^{q}$ is bounded 
uniformly in $\mathbf{X} \in \mathscr{X}_{\Delta(i)^{c}}^{\cdot(q)}$. Since $N_{i}^{2}$ is the supremum of functions in $\mathscr{S}$, we conclude in particular that $\int N_{i}^{2} d Q<\infty$. Hence $Q$ is of second order.

Step 4. Verification of the Gibbs property. To show that $Q$ is a continuum Potts measure we need to check that, for any $k \geq 0$ and any local function $f$ on $\mathscr{x}^{\circ}(q)$ with $0 \leq f \leq 1$,

$$
\int f d Q=\int f_{\Lambda(k)} d Q
$$

Here we use the abbreviation $f_{\Lambda}(\mathbf{X})=\int f d Q_{\Lambda \mid \mathbf{X}}, \mathbf{X} \in \mathscr{X}_{\Lambda^{c}}^{\cdot(q)}$. For notational convenience we confine ourselves to the case $k=0$; for general $k$ one can argue similarly. Since $f \in \mathscr{Y}$, we know that $\int f d Q=\lim \int f \bar{Q}_{n^{\prime}}$ along the subsequence $\left(n^{\prime}\right)$ with $\bar{Q}_{n^{\prime}} \rightarrow Q$ The consistency and translational homogeneity of the Gibbs distributions imply that, for each $n$,

$$
\int f d \bar{Q}_{n}=v_{n}^{-1} \sum_{i \in L(n)} \int\left(f \circ \vartheta_{\imath}\right)_{\Delta(\imath)} d Q_{n}=\int f_{\Delta(0)} d \bar{Q}_{n}
$$

Therefore it only remains to show that

$$
\int f_{\Delta(0)} d Q=\lim \int f_{\Delta(0)} d \bar{Q}_{n^{\prime}}
$$

along the subsequence $\left(n^{\prime}\right)$. This is obvious when $\psi$ has finite range because then $f_{\Delta(0)}$ is local In the general case, we approximate $f_{\Delta(0)}$ by the bounded local functions $f_{\Delta(0), k}=f_{\Delta(0)}(\cap \Lambda(k)), k \geq 1$. Namely, arguing as in the first lines of the proof of Lemma 7.4 of [11] we find that

$$
\begin{aligned}
\left|f_{\Delta(0)}(\mathbf{X})-f_{\Delta(0), k}(\mathbf{X})\right| \leq & 2 \sum_{\jmath \in \mathbb{Z}^{d} \backslash \Lambda(k)} \Psi_{j} N_{j}(\mathbf{X}) \\
& \times\left[\int N_{0} d Q_{\Delta(0) \mid \mathbf{X}}+\int N_{0} d Q_{\Delta(0) \mid \mathbf{X} \cap \Lambda(k)}\right]
\end{aligned}
$$

for sufficiently large $k$ In addition to our assumptions $\varphi \geq 0$ and (14), this estimate requires only the upper regularity of $\varphi+\psi$, which means that

$$
\sum_{x \in X \cap \Delta(i), y \in X \cap \Delta(j)}(\varphi+\psi)(x-y) \leq \Psi_{i-\jmath} N_{i}(X) N_{\jmath}(X)
$$

for all $X \in \cdot X^{*}$ whenever $|i-j|$ exceeds some constant. This property follows trivially from (A2) and (A4). If $\psi$ is superstable, a variant of Lemma 7.3 in [11] shows that there exists a constant $C<\infty$ such that

$$
\int N_{0} d Q_{\Delta(0) \mid \mathbf{X}} \leq C+D \sum_{i \neq 0} \Psi_{i} N_{i}(\mathbf{X})
$$

with $D=2 / a$. If $\psi \geq 0$, we can even set $D=0$. Inserting this into (19) and using the Cauchy-Schwarz inequality and (17) we arrive at the estimate

$$
\int\left|f_{\Delta(0)}-f_{\Delta(0), k}\right| d \bar{Q}_{n} \leq 4\left[C m^{1 / 2}+D \Psi m\right] \sum_{j \in \mathbb{Z}^{d} \backslash \Lambda(k)} \Psi_{j}=. \varepsilon(k)
$$


The same estimate holds with $Q$ in place of $\bar{Q}_{n}$. Since $\varepsilon(k) \rightarrow 0$ as $k \rightarrow \infty$ and $f_{\Delta(0), k} \in \mathscr{L}$ for all $k$, assertion (18) follows immediately. The proof of Proposition 4.1 is therefore complete.

We conclude with two remarks.

Remark 4.2. Proposition 4.1 evidently implies the existence of continuum Potts measures for arbitrary activities $z>0$. Of course, it is also true in the case $q=1$ and then gives us the existence of tempered Gibbs measures for pair interactions $\psi$ satisfying (A3) and the upper regularity (20) (with $\varphi \equiv 0$ ), as established first by Dobrushin [5] and Ruelle [30]. Compared with [30], the preceding argument is less elementary but also much less involved, whereas the methods of [5] need a non-integrable singularity of $\psi$ at the origin. Note, however, that these references provide stronger information on the moments of the limiting Gibbs measures.

Remark 4.3. Suppose each particle type $s$ is equipped with its own activity $z_{s}>0$, so that the parameter $z>0$ is replaced by the vector $\mathbf{z}=\left(z_{1}, \ldots, z_{q}\right)$. Let $Z_{\Lambda \mid \mathbf{Y}}(\mathbf{z})$ be the associated partition function, cf. (1). Using the techniques of [10], one can show that the pressure

$$
p(\mathbf{z})=\lim _{n \rightarrow \infty} v_{n}^{-1} \log Z_{\Lambda(n) \mid \mathbf{Y}(n)}(\mathbf{z})
$$

(exists and) is equal to

$$
-\inf _{R}\left[U(R)+I_{1}(R)-\sum_{s=1}^{q} \rho_{s}(R) \log z_{s}\right] .
$$

In the above, the infimum extends over all probability measures $R$ on $\mathscr{X}^{(q)}$,

$$
U(R)=\lim _{n \rightarrow \infty} v_{n}^{-1} \int H_{\Lambda(n) \mid \mathbf{Y}(n)} d R_{(n)}
$$

is the energy density, and $I_{1}(R)$ is the relative entropy density of $R$ with the Poisson point processes of intensity 1 as reference measures. If $z$ is so large as required before and $\mathbf{z}=(z, \ldots, z)$, one can further deduce from (16) with the methods of [10] that the infimum above is attained by $\bar{Q}$ and thus also by $Q_{1}, \ldots, Q_{q}$. (In fact, if $\psi$ has a non-integrable singularity at the origin, an analog of the variational principle in [11] shows that the infimum is attained precisely for all $\left(\vartheta_{x}\right)_{x \in \mathbb{R}^{d}}$-invariant continuum Potts measures.) Since $\rho_{s}\left(Q_{s}\right)>\rho_{s}\left(Q_{t}\right)$ for $1 \leq s \neq t \leq q$, this means that the convex function $\left(\log z_{1}, \ldots, \log z_{q}\right) \rightarrow p(\mathbf{z})$ admits $q$ tangent hyperplanes at $(\log z, \ldots, \log z)$ with distinct slopes in each direction. Consequently, the pressure $p(\mathbf{z})$ is not differentiable with respect to $z_{s}$ at $(z, \ldots, z)$ for each $1 \leq s \leq q$, provided $z$ is large enough. By the usual Legendre-Fenchel duality formalism, this non-differentiability corresponds to a discontinuity of the pressure as a function of the particle densities $\rho_{1}, \ldots, \rho_{q}$.

\section{References}

1 Bollobas, B (1985): Random graphs London etc: Academic Press

2 Bricmont, J, Kuroda, K, and Lebowitz, J L (1984): The structure of Gibbs states and coexistence for non-symmetric continuum Widom-Rowlinson models $Z$ Wahrscheinlichkeitstheorie verw Geb 67, 121-138 
3 Chayes, J T, Chayes, L and Kotecky, R (1995): The analysis of the Widom-Rowlinson model by stochastic geometric methods Commun Math Phys 172, 551--569

4 Dobıushin, R L (1970): Prescribing a system of random variables by conditional distributions $T h$ Probab Appl 15, 458-486

5 Dobrushın, RL (1970): Gibbsian ıandom fields for particles without hard core Theor Math Phys 4, 705-719

6 Dobrushın, R L and Minlos, R A (1967): Existence and continuity of pressure in classical statistical physics Th Probab Appl 12, 535-559

7 Edwards, R G and Sokal. A D (1988): Generalization of the Fortuin-Kasteleyn-Swendsen-Wang 1epresentation and Monte Carlo algorithm Phys Rev D 38, 2009-2012

8 Fortuin, C M and Kasteleyn, P W (1972): On the random-cluster model I Introduction and relation to other models Physica 57, 536-564

9 Georgii, H -O (1988): Gibbs Measures and Phase Transitions New York: de Gruyter

10 Georgii, H -O (1994): Large deviations and the equivalence of ensembles for Gibbsian particle systems with superstable interaction Probab Th Rel Fields 99, 171-195

11 Georgii, H-O (1995): The equivalence of ensembles for classical systems of particles $J$ Statist Phys 80, 1341-1378

12 Georgii, H -O and Küneth, T (1995) Stochastic comparison of point random fields Preprint

13 Georgii, $\mathrm{H}-\mathrm{O}$ and Zessin, H (1993): Large deviations and the maximum entropy principle for marked point 1andom fields Probab Th Rel Fields 96, 177-204

14 Giacomin, G, Lebowitz, J L and Maes, C (1995): Agreement percolation and phase coexistence in some Gibbs systems J Statist Phvs 80, 1379-1403

15 Given, J A and Stell, G (1990): The Kirkwood-Salsburg equations for continuum percolation $J$ Statist Phys 59, 981-1018

16 Grimmett, G (1994): Percolative problems In: Probability and Phase Transition (ed G Grimmett), Dordrecht: Kluwer, pp 69-86

17 Israel, R B (1979): Convexity in the Theorv of Lattice Gases Princeton, NJ: Princeton University Piess

18 Johansson, K (1991): Separation of phases at low temperatures in a one-dimensional continuous gas Commun Math Phys 141, 259-278

19 Johansson, K (1995): On separation of phases in one-dimensional gases Commun Math Phvs 169, $521-561$

20 Kallenberg, O (1983): Random measures 3rd edition Berlin: Akademie Verlag

21 Kesten, H (1982): Percolation Theory for Mathematicians Boston etc: Birkhäuse1

22 Klein, W (1982): Potts-model formulation of continuum percolation Phys Rev' B 26, 2677-2678

23 Lebowitz, JL and Lieb, EH (1972): Phase transition in a continuum classical system with finite interactions Phys Lett 39A, 98-100

24 Matthes, K, Kerstan, J and Mecke, J (1978): Infinitely Divisible Point Processes Chichester: Wiley

25 Meester, R and Roy, R (1996): Continuum Percolation Cambridge: Cambridge University Press

26 Meester, R and Steif, J (1994): Personal communication

27 Minlos, R A (1967): Limiting Gibbs distribution Funct Anal Appl 1, 140-150\&206-217

28 Penıse, M D (1991): On a continuum peicolation model Adv Appl Probab 23, 536-556

29 Preston, C J (1977): Spatial birth-and-death processes Bull Int Statist Inst 46(2), 371-391

30 Ruelle, D (1970): Superstable interactions in classical statistical mechanics Commun Math Phys 18, 127-159

31 Ruelle, D (1971): Existence of a phase tiansition in a continuous classical system Phys Rev Lett 27, 1040-1041

32 Widom, B and Rowlinson, J S (1970): New model for the study of liquid-vapor phase transition $J$ Chem Phys 52, 1670-1684

\section{Communicated by Ya.G. Sinai}

